\title{
Explaining variation in the burden of child and adolescent tuberculosis
}

From the authors:

We read with interest the recent correspondence from CHEN et al. [1] regarding the burden of notified tuberculosis (TB) among adolescents and young adults in Taiwan. The authors report that 10-24 year olds constituted only $5.2 \%$ of all newly diagnosed TB patients in Taiwan from 2005 to 2016, and that this percentage declined from around 6\% in 2005 to around 4\% in 2016. They contrast these figures with our estimate that young people constituted $17 \%$ of all people developing incident TB globally in 2012 [2]. There are several factors that explain this apparently large discrepancy.

First, our figure of $17 \%$ is a global average, covering settings that are demographically and epidemiologically diverse. The age distribution of TB in any setting depends on three factors: the age structure of the population, the intensity of TB transmission, and the success of TB prevention efforts in young age groups. The proportion of TB cases which affect young people will be lower than average in less intense TB epidemics, in countries with older populations, and in countries with more successful prevention programmes. At one extreme, in Western Europe, most nations have TB incidence below 20 cases per 100000 person-years [3], and young people constitute only $14-18 \%$ of the total population [4]. At the other extreme, in southern Africa estimated TB incidence exceeds 300 per 100000 person-years [3] and fully one third of the population are aged 10-24 years [4], resulting in an epidemic concentrated among those in young and middle adulthood (figure 1). In Taiwan, young people constitute around $16 \%$ of the population, similar to Europe and other high income settings [5], and as noted by the authors, reported TB incidence is 44 cases per 100000 person-years. As such, it is to be expected that the burden of disease among young people in Taiwan is below the global average.

Furthermore, high rates of TB transmission have a disproportionate impact on children and adolescents [6]. As TB incidence declines, the burden of disease shifts into progressively older age groups, reflecting a greater burden due to reactivation of infection acquired in periods of higher transmission early in life [7]. Chen et al. [1] describe impressive declines in reported TB in Taiwan, from 73 cases per 100000 personyears in 2005 to 44 per 100000 person-years in 2016, a decline of approximately $3.6 \%$ per year. Consequently, it is not surprising that the proportion of disease among young people has declined at the same time, and that there were greater declines in TB among young people than older people. This is consistent with observations in Japan during a period of substantial decline in TB incidence [7], and with other countries in the Western Pacific region (figure 1).

Secondly, there will usually be a discrepancy between notification data, which show only diagnosed and reported cases, and estimates of all incident disease which account for imperfect case detection (refer to the white gaps in figure 1) [3]. Evidence is emerging that young people with TB may be at higher risk of misdiagnosis and under-detection than adults [8]. This is consistent with the reality that in most settings, young people have reduced access to health services due to limited financial resources, the demands of school and work, family control over their ability to seek care, and lower health literacy than adults [9]. Our estimates were for all incident TB, including undiagnosed and unreported TB [2]. Consequently, our estimates are higher than what is observed in notification data, because we adjusted for the case detection gap.

CHEN et al. [1] describe a strong TB control response in Taiwan over recent decades, including an expansion of contact tracing to cover almost $100 \%$ of close contacts in 2017 [1]. This is a major achievement. We would add a note of caution, however, in response to the assertion that "young patients [...] may have

@ERSpublications

The burden of tuberculosis in children and young people depends greatly on the intensity of the TB epidemic, the demographics of the population, and the success of prevention efforts http://bit.ly/2ETFTik

Cite this article as: Snow KJ, Sawyer SM, Denholm JT, et al. Explaining variation in the burden of child and adolescent tuberculosis. Eur Respir J 2019; 53: 1901007 [https://doi.org/10.1183/13993003.010072019]. 

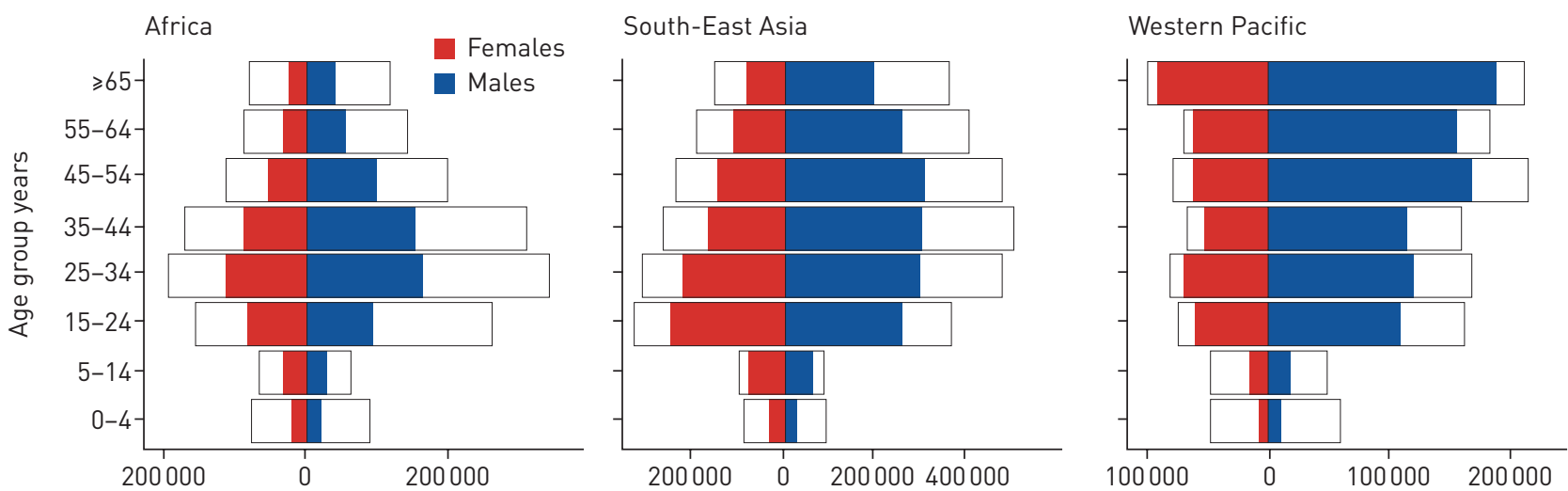

FIGURE 1 Estimated burden of disease (outlines) and case notifications (female in red and male in blue) by age and sex in Africa, South-East Asia and Western Pacific region. Reproduced with permission from the World Health Organization Global Tuberculosis Report 2018 [3].

compromised treatment adherence in some areas. Therefore, adolescents and young adults with TB clearly benefit more from the [directly observed treatment, short course] programme." Medication adherence is a well-recognised challenge for adolescents with any chronic health condition, including TB, due to complex developmental and health system factors [9]. However, the traditional approach of clinic-based direct observation of therapy (DOT) can majorly disrupt participation in education and employment, which will already be threatened by the diagnosis of a stigmatised condition like TB. Unless health clinics are co-located or close to schools, adolescents who are required to attend a daily clinic will miss many hours of education, a risk factor for poor learning, disengagement from schooling and early school leaving. The World Health Organization has de-emphasised clinic-based DOT in the End TB Strategy [10], and is encouraging TB services to develop and implement patient-centred models of care. Young people with TB may indeed require more intensive support to achieve consistent adherence to medications, especially with second-line drugs or when complex comorbidities such as HIV are present, and if they are poorly supported by their families. However, this care should be delivered in ways that respect their developing autonomy, their desire for privacy, and the importance of their continued engagement in education or employment. We in the TB community have much to learn from colleagues in HIV and other chronic disease services with regard to providing high-quality, patient-centred care to young people with TB.

Kathryn J. Snow $\oplus^{1}$, Susan M. Sawyer ${ }^{2}$, Justin T. Denholm ${ }^{3}$ and Stephen M. Graham $\odot^{1}$

${ }^{1}$ Centre for International Child Health, Dept of Paediatrics, University of Melbourne, Melbourne, Australia. ${ }^{2}$ Centre for Adolescent Health, The Royal Children's Hospital, Melbourne, Australia. ${ }^{3}$ Victorian Tuberculosis Program, Melbourne Health, Melbourne, Australia.

Correspondence: Kathryn Snow, University of Melbourne, Dept of Paediatrics, Level 2 East, Royal Children's Hospital, 50 Flemmington Rd, Parkville, Victoria 3052, Australia. E-mail: kathryn.snow@unimelb.edu.au

Received: May 202019 | Accepted after revision: May 292019

Conflict of interest: None declared.

\section{References}

1 Chen Y-Y, Pan S-W, Shen H-S, et al. Declining trend in incidence of tuberculosis in adolescents and young adults in taiwan. Eur Respir J 2019; 53: 1801305.

2 Snow K, Sismanidis C, Denholm J, et al. The incidence of tuberculosis among adolescents and young adults: a global estimate. Eur Respir J 2018; 51: 1702352.

3 World Health Organization. Global Tuberculosis Report 2018. Geneva, World Health Organization, 2018.

4 United Nations Population Division. World Population Prospects, 2017 Revision. 2017. Available from: http://esa. un.org/unpd/wpp/DVD/

5 Central Intellegence Agency. World Factbook. 2019. https://www.cia.gov/library/publications/the-world-factbook Date last accessed: May 20, 2019.

6 Marais BJ, Gie RP, Schaaf HS, et al. The clinical epidemiology of childhood pulmonary tuberculosis: A critical review of literature from the pre-chemotherapy era. Int J Tuberc Lung Dis 2004; 8: 278-285.

7 Mori T, Leung CC. Tuberculosis in the global aging population. Infect Dis Clin North Am 2010; 24: 751-768.

8 Department of Health, Republic of the Philippines. Provisional NTPS 2016 Technical Report. Manila, Foundation for the Advancement of Clinical Epidemiology, 2017.

9 Patton GC, Sawyer SM, Santelli JS, et al. Our future: a Lancet commission on adolescent health and wellbeing. Lancet 2016; 387: 2423-2478.

10 World Health Organization. End TB Strategy. Geneva, Global TB Programme, 2015. 